\title{
A Knowledge Management System for the Variation in Regional Clinical Pathways of Traditional Chinese Medicine Based on Smart Cloud Services
}

\author{
Xiaoying Hu, Jiali Liu, Shuwu Li, Kai Li* \\ Zhejiang Chinese Medical University, Hangzhou 310053, China
}

Corresponding Author Email: kaili@fudan.edu.cn

https://doi.org/10.18280/ria.330602

Received: 10 May 2019

Accepted: 18 September 2019

\section{Keywords:}

clinical pathways (CPs), variation management, knowledge management system (KMS), cloud services

\begin{abstract}
This paper aims to provide an effective solution to the variation in clinical pathways (CPs) of traditional Chinese medicine (TCM). First, a semi-structured interview was carried out among stakeholders, such as system research and design (R\&D) engineers, Chinese medicine hospital managers, medical staff, patients and health bureau officials. Their demand on the knowledge management system (KMS) for TCM CPs variation was summarized through the interview. In the light of the user demand, the author designed a novel KMS for variation in regional TCM CPs (KMS-TCMCPV) based on smart cloud services. The proposed system consists of four modules: variation warning service, variation intervention service, patient-oriented smart service, and smart cloud service. The architecture and implementation of each service were explained in details. The proposed system effectively overcomes the defects of the previous system, such as the lack of variation warning, the limited feedback channel, and the inability to share empirical knowledge. Our system successfully enhances the diagnosis and treatment of Chinese medicine hospitals, and improves the patient satisfaction.
\end{abstract}

\section{INTRODUCTION}

Clinical pathways (CPs) are sequences of standardized clinical care provided to patients with specific medical problems and a predictable clinical course. The CPs offer an effective and popular hospital management tool that improves the quality, controls the cost and optimizes the process of medical services [1-3].

In traditional Chinese medicine (TCM), the CPs implementation is incorporated with therapies, techniques and equipment with TCM characteristics, aiming to streamline the diagnosis and treatment plan [4]. Therefore, the TCM CPs need to effectively combine syndrome differentiation and disease differentiation. This pushes up the variation rate of the CPs, suppressing the completion rate, calling for more knowledge support of variation control [5].

In the TCM, the CPs variation means some patients may deviate from the planned CP flow, due to the differences in hospital environment, medical skills and patient conditions [68]. To control the variation, the solution should be designed according to the specific source of the variation, without interrupting the medical care [9]. However, there are complex reasons for the variation in TCM CPs, making it difficult to warn the variation in advance [10].

To date, much attention has been paid to the knowledge management of the variation in the TCM CPs in a single hospital or department $[11,12]$, yielding fruitful results. The variation law of the TCM CPs could be discovered by mining the objective indices (e.g. gender, age, drug dosage, and nursing grade) of data sources like hospital information system (HIS) [13] and electronic medical records (EMR), laying the basis for early warning of the variation [14-16]. Nevertheless, there is little report on the knowledge management of the variation in the TCM CPs from the regional perspective.
Under the background of regional medical integration, it is of great significance to build a knowledge management system (KMS) based on the variation in regional TCM CPs, which helps to optimize regional medical quality and enhance patient satisfaction. Unfortunately, the regional medical institutions cannot effectively prewarn or respond to CPs variation, under the existing KMS for the variation in regional TCM CPs. This is because the knowledge resources are highly dynamic in the Internet environment. What is worse, the competent authority fails to supervise the variation management of the TCM CPs in real time [17].

A possible solution is to design and deploy the KMS based on cloud computing. Cloud computing is a new computing technology based on the Internet. It fully utilizes the emerging techniques like distributed processing, parallel processing and grid computing. In fact, cloud computing has been adopted by many medical institutions to improve the functions of the KMS, including high-performance computing, robust information processing, flexible resource acquisition and expansion, massive data storage, and large-scale cross-domain service scheduling [18].

In this era of big data, cloud services have become increasingly important and prevalent in various applications [19-21]. As shown in Figure 1, the typical architecture of cloud services [22] consists of server clusters, database (DB) clusters (cloud storage), template engines, etc. The dataset is transferred from each server node to the DB cluster, and analyzed and processed on the server cluster.

In light of the above, this paper designs a KMS for regional TCM CPs variation (KMS-RTCMCPV), which supports realtime exchange of health data between terminals and clouds, and pushes the relevant knowledge to all types of users via terminal devices and the Internet. 


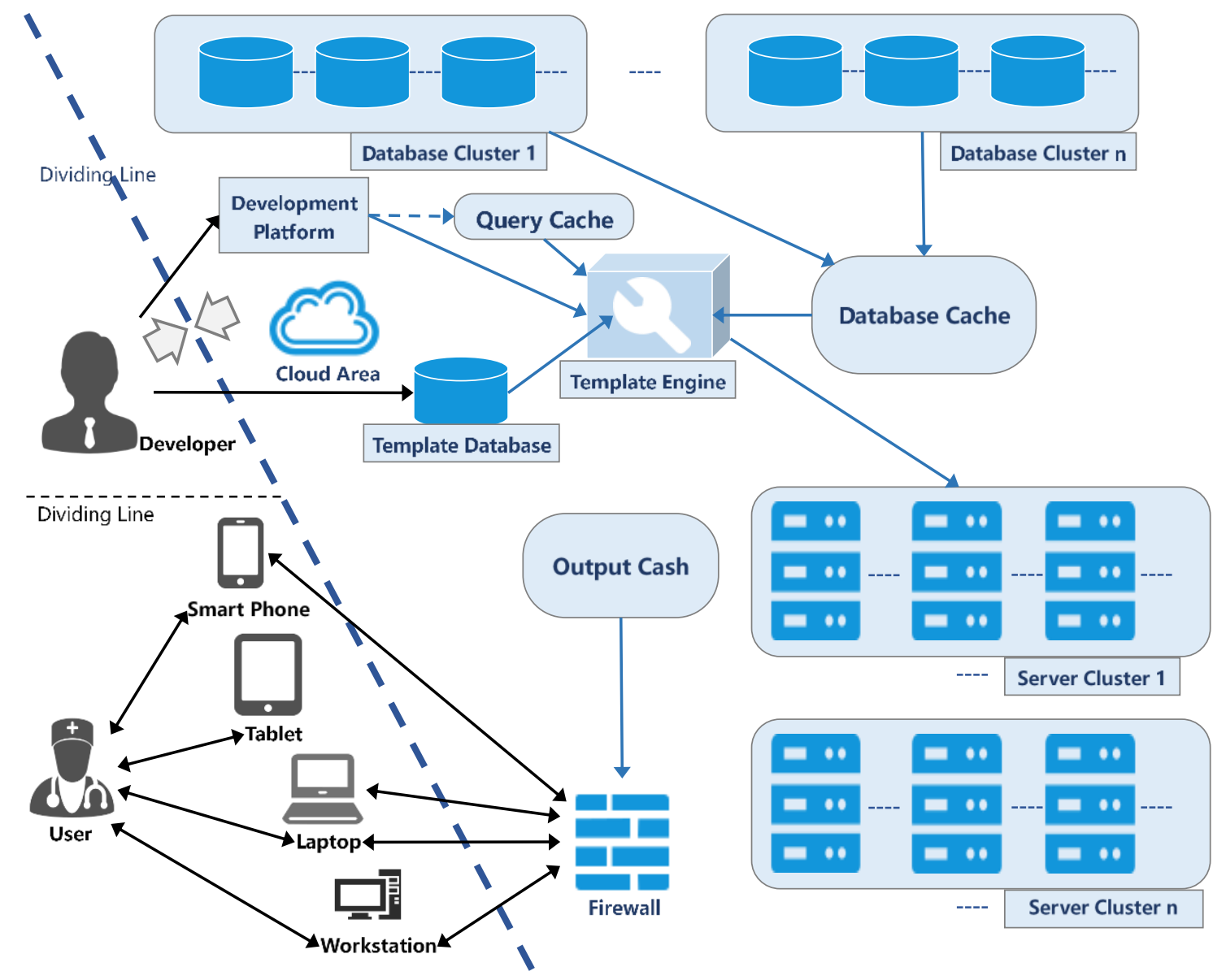

Figure 1. The typical architecture of cloud services

\section{SYSTEM CONSTRUCTION}

\subsection{Semi-structured interview}

The semi-structured interview was selected as the way to identify the user demand for the KMS-RTCMCPV. A total of semi-structured interviews was conducted with system research and design (R\&D) engineers (22\%), Chinese medicine hospital managers (14\%), medical staff $(32 \%)$, patients (24\%) and health bureau officials (8\%). The interview outline is shown in Table 1. The composition of the interviewees is illustrated in Figure 2. The results of the interview are summarized in Table 2.

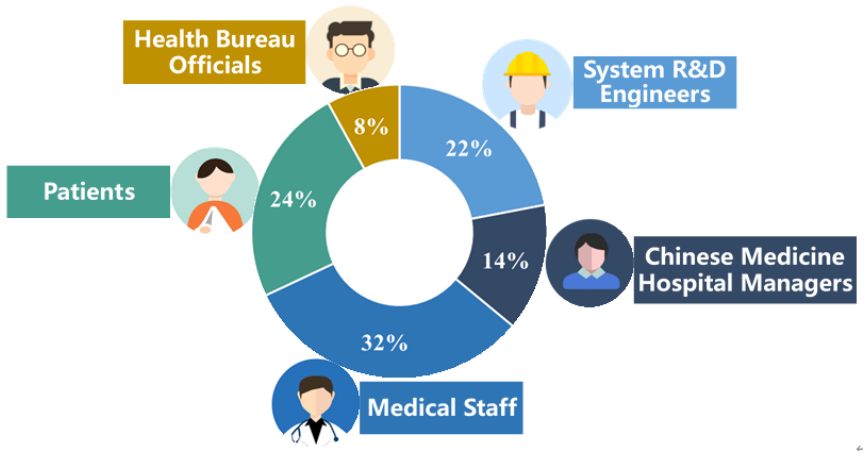

Figure 2. Composition of interviewees

Table 1. The interview outline

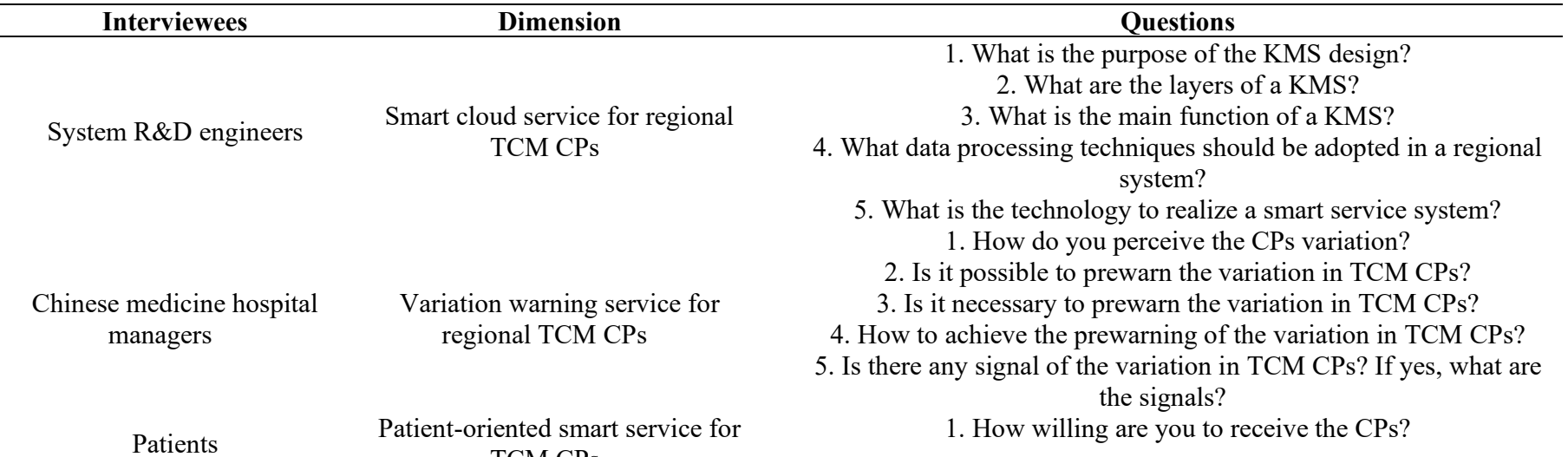


Medical staff

Health bureau officials
Variation intervention knowledge service for regional TCM CPs

Evaluation indices for regional TCM CPs
2. Are you concerned about the implementation of the TCM CPs? If yes, what are your concerns?

3. What services do you expect from the doctor during the implementation of the TCM CPs?

4. What do you know about the TCM CPs?

5 . What do you think of the patient feedback channels?

1. What are your needs in the knowledge management of the TCM CPs?

2. What are the needs of medical students in knowledge management of the TCM CPs?

3. What kind of help do you need to handle the variation caused by the medical staff?

4. What kind of help do you need to handle the variation caused by the equipment?

5. What kind of help do you need to handle the variation caused by the hospital system?

1. How about making patient satisfaction an evaluation index of regional TCM CPs?

2. How about making doctor satisfaction an evaluation index of regional TCM CPs?

3. How about making the average length of stay an evaluation index of regional TCM CPs?

4. How about making the variation rate an evaluation index of regional TCM CPs?

5.Is it necessary to monitor the TCM CPs in real time?

Table 2. Interview results

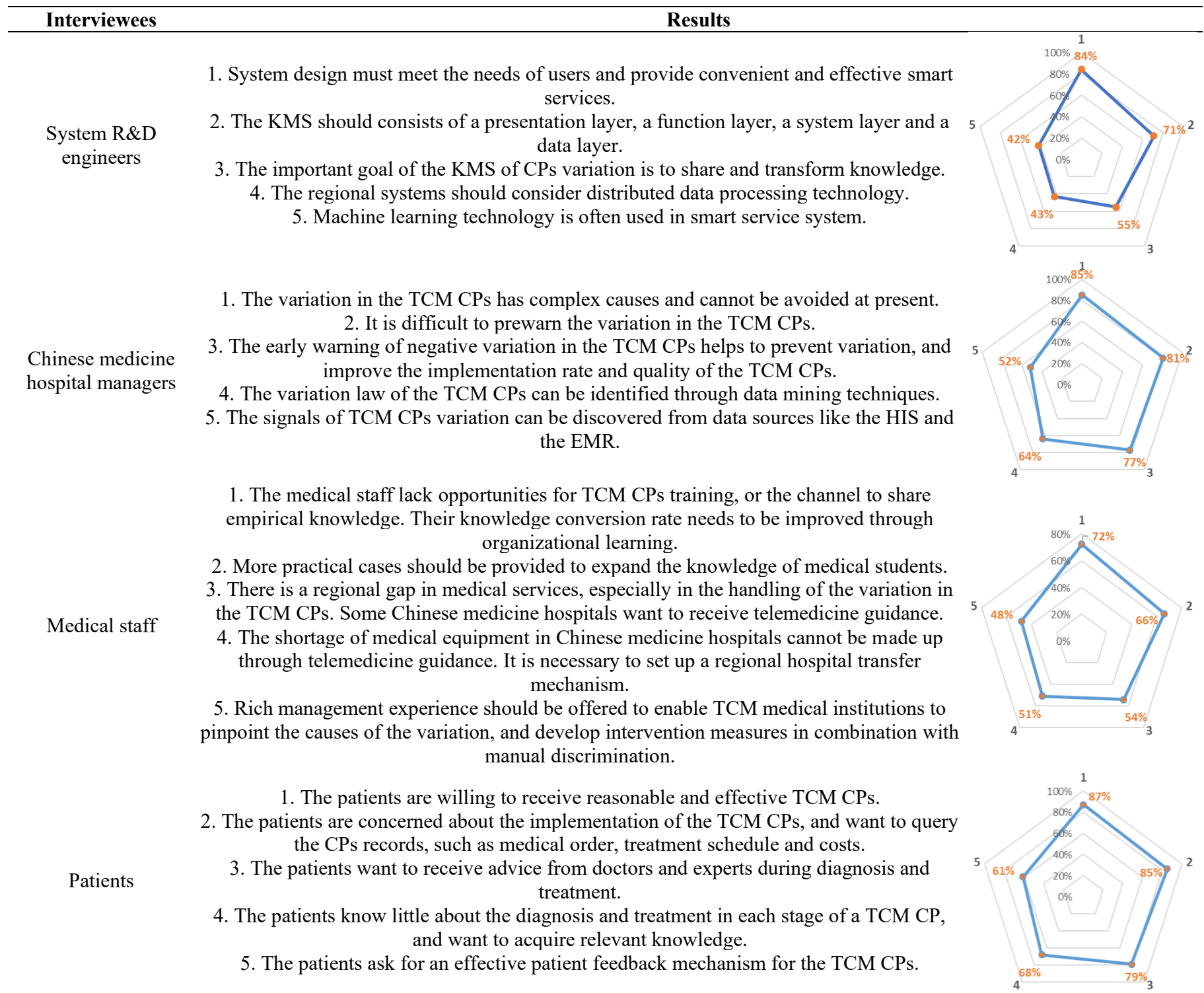


1. Patient feedback is important to quality evaluation of regional TCM CPs.

2. Doctor satisfaction is important to quality evaluation of regional TCM CPs

3. The average length of stay is covered in the evaluation index called regional TCM CPs

Health bureau officials efficiency.

4. Both cost control and variation rate should be considered to evaluate the quality of regional TCM CPs.

5. Real-time monitoring promotes the scientific and timely management of the TCM CPs

The early warning and control of the TCM CPs in departments help to prevent empirical management and reduce the inertia of operations.

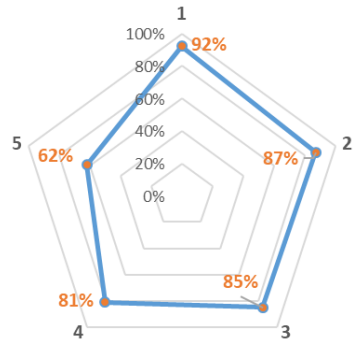

The interviews reveal some defects with the current KMSRTCMCPV. These defects can be overcome by unifying the CPs process data with CPs knowledge, and optimizing the variation warning service and knowledge sharing mechanism for the TCM CPs. Therefore, this paper aims to design a KMS that can monitor the data on regional TCM CPs in real time, assist the medical staff in the variation intervention for the TCM CPs, provide them with smart knowledge service about the TCM CPs, and highlight the treatment experience of patients. Figure 3 shows the service modules of our system and their relationships with the needs raised by the interviewees.

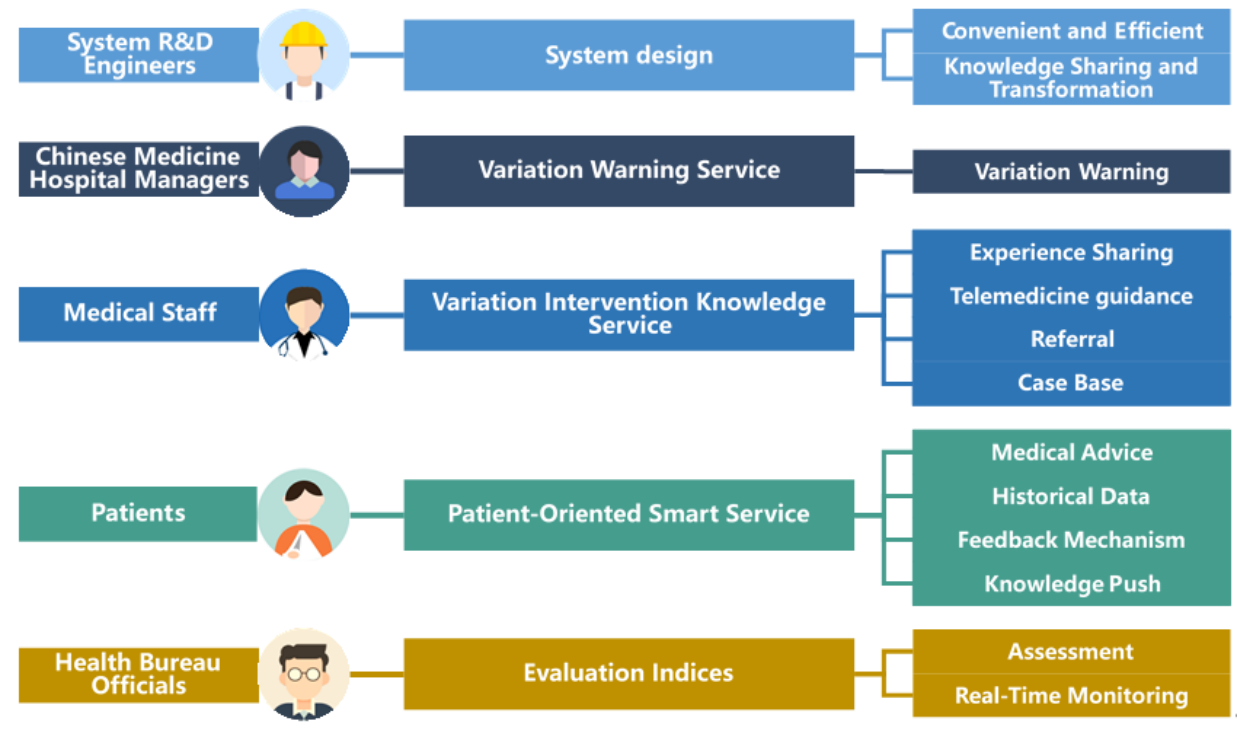

Figure 3. Service modules and the needs raised by the interviewees

\subsection{Construction of the KMS-RTCMCPV}

As mentioned above, this paper aims to design a KMSRTCMCPV based on cloud services, and rely on the system to realize real-time data exchange and smart knowledge sharing for regional TCM CPs. The system architecture is presented in Figure 4. There are four modules of the system: variation warning service, variation intervention service, patientoriented smart service, and smart cloud service.

\section{APPLICATION AND IMPLEMENTATION OF CLOUD SERVICES IN THE KMS-RTCMCPV}

\subsection{Variation warning service}

\subsubsection{Service contents}

Out of the various research directions of TCM CPs variation, this paper chooses the knowledge management of CPs variation as the optimization strategy, due to its applicability to most medical staff. The variation in TCM CPs mainly come from the following sources: medical staff [23] (e.g. the misalignment of medical orders, maloperation of information system, as well as differences in technical level, work attitude and communication ability); patients [8] (e.g. mental state and subjective needs); disease outcome [24] (e.g. treatment of hospital complications, management of preoperative complications, and disease complexity); hospital system [25] (e.g. departmental coordination, equipment deployment, and rules on leave). The variations can be divided into positive and negative categories based on their nature [26].

The complex causes of TCM CPs variation make it difficult to give early warning. Therefore, our KMS-RTCMCPV mines the variation in regional TCM CPs, and determines the association rules between the variation and the objective indices of data sources like the HIS and the EMR, creating a variation knowledge database. Through intelligent calculation, the manager of the Evaluation Center for regional TCM CPs prepares the texts of the knowledge on the regional TCM CPs to be tested and that on the regional TCM CPs that have variated. After the texts are analyzed, a pathway will be marked if it has the risk of variation, and then the variation intervention service will be activated. The variation warning service will send a list of early warning data to Chinese medicine hospitals, departments and medical staff, and push countermeasures and related experts, which are determined through intelligent computing and matching. The operation of variation warning service is displayed in Figure 5.

As shown in Table 3, each warning of variation contains several types of information, including the ID number of the marked CP, the ICD-10 code, as well as the stage, type, cause and time of the variation. 


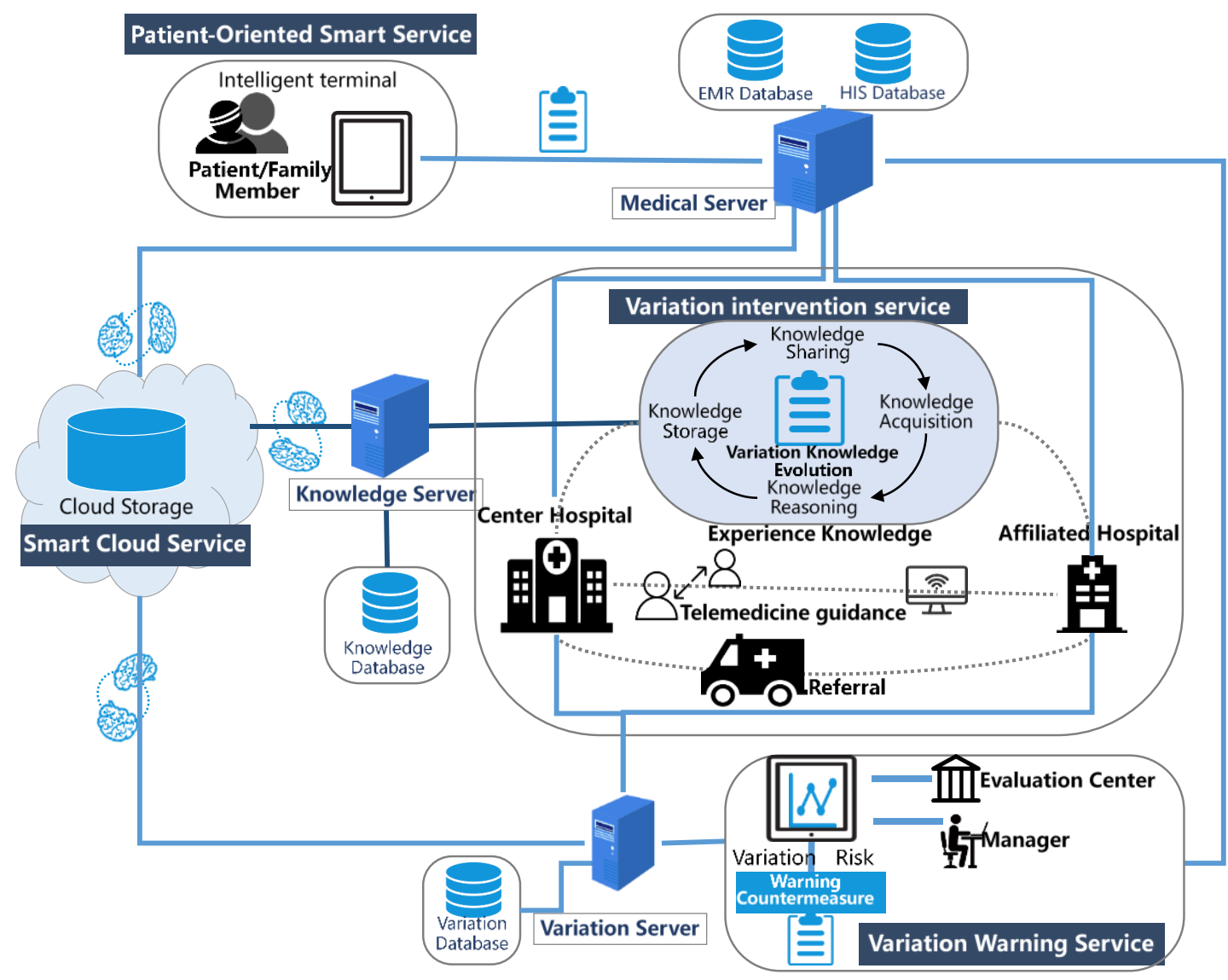

Figure 4. The architecture of our KMS-RTCMCPV

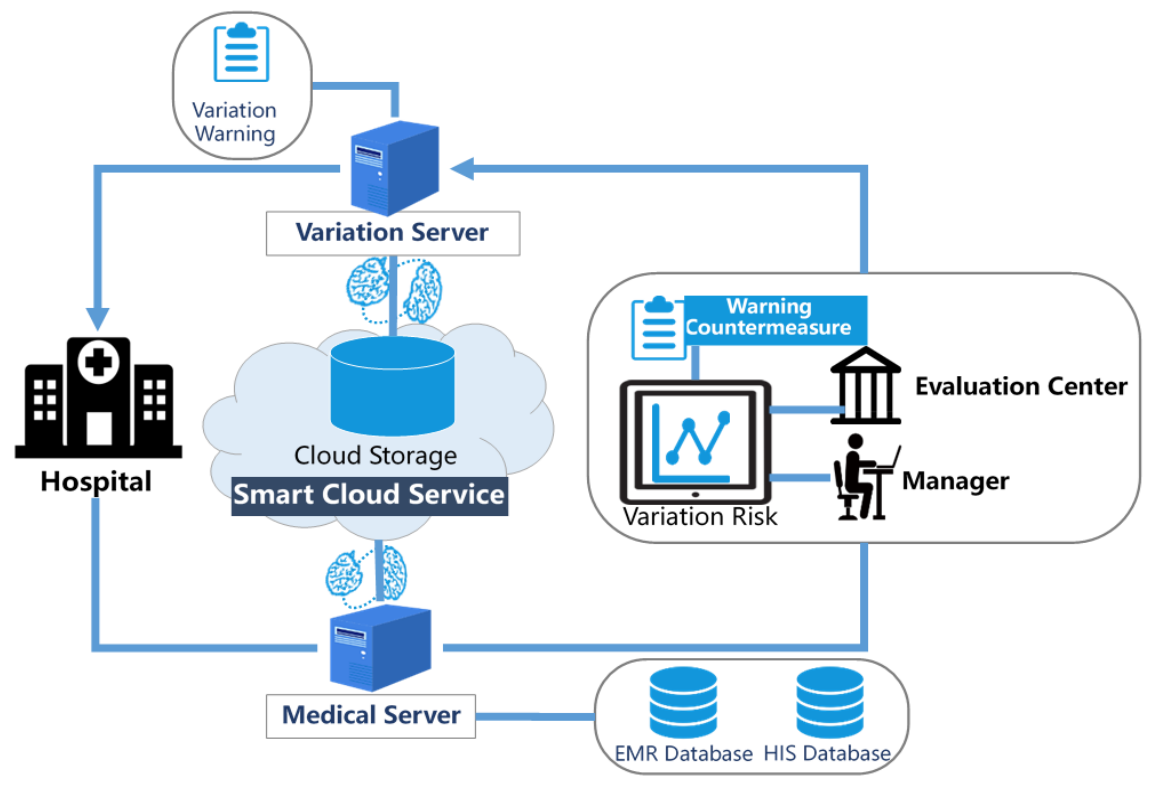

Figure 5. The operation of variation warning service

Table 3. The information contained in each variation warning

\begin{tabular}{ccccc}
\hline No. & Name & Type & Description & Constraint \\
\hline 1 & CPV_Index & Int & Number & Non-empty \\
2 & CPV_Num & DR & ID number, primary key & Non-empty \\
3 & CPV_ICD-10 & DR & ICD-10 & Non-empty \\
4 & CPV_Epiosde & DR & Variation process & Non-empty \\
5 & CPV_Type & DR & Variation type & Non-empty \\
6 & CPV_Reason & DR & Variation cause & Non-empty \\
7 & CPV_Dtime & DateTime & Variation time & Non-empty \\
\hline
\end{tabular}




\subsubsection{Service implementation}

The variation warning service monitors the TCM CPs variation based on the EMR, the HIS and the variation knowledge database. Once the data deviate from the standard for the pathway, the monitored pathway will be marked and its variation information will be stored in the system.

In our system, the intelligent warning is realized by text mining. The CPs with high variation risks can be screened out through the calculation of the knowledge $K_{i}$ on the TCM CPs to be tested and that $K_{j}$ on the variated TCM CPs. The knowledge is represented in Extensible Markup Language (XML). The closeness between $K_{i}$ and $K_{j}$ can be computed by:

$$
\operatorname{sim}_{\text {text }}\left(K_{i}, K_{j}\right)=\cos \left(K_{i}, K_{j}\right)=\frac{\sum_{i} K_{i} K_{j}}{\sqrt{\sum_{j} K_{j}^{2} \sum_{i} K_{i}^{2}}}
$$

\subsection{Variation intervention service}

\subsubsection{Service content}

Through investigation, it is learned that the same TCM CP varies at different rates in different Chinese medicine hospitals, requiring different coping mechanisms. The difference arises because each hospital has its unique hospital system, medical level and equipment deployment. In light of the dynamic environment, this paper develops a variation intervention service suitable for all medical staff in the same region to effectively suppress TCM CP variation.

Based on the variation warning service, the variation intervention service relies on knowledge evolution technology to share a massive amount of CPs intervention knowledge across the regional information platform. In this way, all medical staff in the region can improve their medical level, and the hospitals on different scales in the region can cooperate with each other. The variation intervention service provides three mechanisms to cope with the variation.

(1) Experience knowledge sharing: If the variation is induced by hospital system and medical staff, the knowledge push method will be adopted to cope with the variation. Our system calculates the closeness between intervention knowledge and medical staff, according to their knowledge performance. Then, the knowledge will be pushed to the medical staff with high closeness. For example, empirical programs for preoperative psychological development of patients will be pushed to Chinese medicine hospitals that frequently face patient-related variations. Our system also provides abundant teaching cases to interns or medical students, which serve as a medical record database for clinical teaching. This database boasts numerous TCM CPs and various kinds of diseases in the region.

(2) Telemedicine guidance: If the variation is induced by the medical staff only, the center hospital in the region will provide remote consultation to the medical staff, in addition to the knowledge push.

(3) Referral: If the variation is induced by the hospital equipment, the system will apply for referral to the center hospital in the region, using the transfer channels. The center hospital has the ability to coordinate resources and conduct technical collaboration with affiliated hospitals.

The operation of variation intervention service is shown in Figure 6.

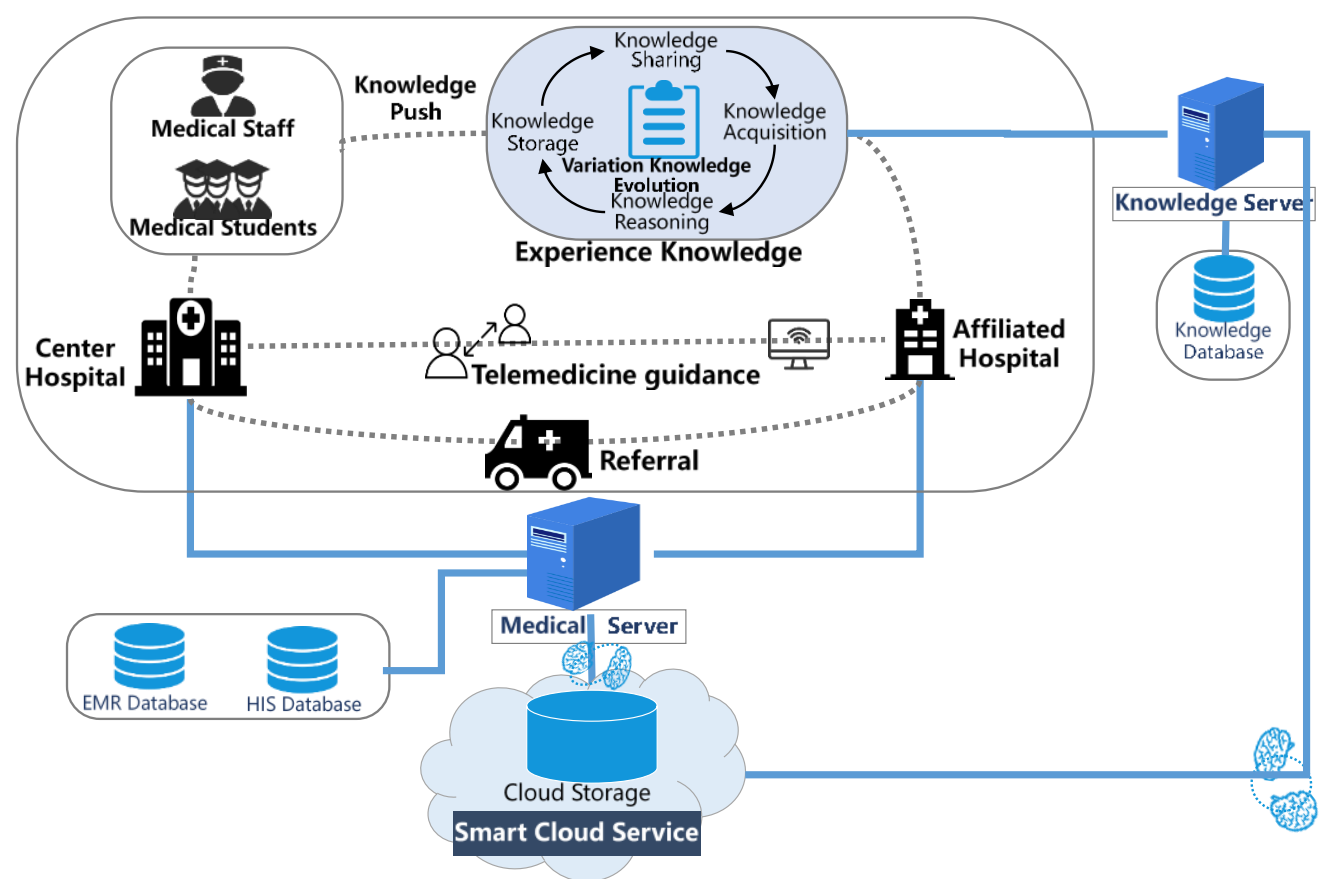

Figure 6. The operation of variation intervention service

\subsubsection{Service implementation}

(1) Matrix design

Source matrix

The source matrix is designed to preserve the source, i.e. the TCM CP, of knowledge $(\mathrm{K})$, laying the basis for computing knowledge closeness:

$C P_{1}$
$K_{1}$
$K_{2}$
$K_{3}$
$K_{4}$$\left[\begin{array}{llll}b_{C P 11} & b_{C P 12} & b_{C P 13} & b_{C P 14} \\ b_{C P 21} & b_{C P 22} & b_{C P 23} & b_{C P 24} \\ b_{C P 31} & b_{C P 32} & b_{C P 33} & b_{C P 34} \\ b_{C P 41} & b_{C P 42} & b_{C P 43} & b_{C P 44}\end{array}\right]$


where, $b_{C P}$ is a Boolean variable about the class of the TCM CPs, which is the source of knowledge. If knowledge $i$ $(i=1,2,3,4)$ comes from the TCM CPs of the same disease, then the value of $b_{C P i j}$ is 1 ; otherwise, the value of $b_{C P i j}$ is 0 . This matrix reflects the knowledge sources, facilitating the retrieval and push of knowledge.

From the perspective of knowledge source (TCM CPs), the closeness $\operatorname{Sim}_{\mathrm{CP}}\left(K_{i}, K_{j}\right)$ between two pieces of knowledge can be computed by:

$$
\operatorname{Sim}_{C P}\left(K_{i}, K_{j}\right)=\frac{\sum_{t=1}^{n_{M}}\left|\left(b_{C P i t}-b_{C P j t}\right)\right|}{2}
$$

If two pieces of knowledge come from the same TCM CP, then their similarity distance is relatively small.

Knowledge representation matrix

Medical staff $\left(M_{1}, M_{2}, M_{3}, \ldots\right)$ could contribute, modify or use knowledge $\left(K_{1}, K_{2}, K_{3}, \ldots\right)$. The times of contribution and the frequency of modification and use were recorded.

Knowledge contribution matrix. If two pieces of knowledge come from the same medical person, then their similarity distance is relatively small. The contribution of the medical person to each piece of knowledge can be expressed as:

$$
\begin{gathered}
M_{1} \\
K_{1} \\
K_{2} \\
K_{3} \\
K_{4}
\end{gathered}\left[\begin{array}{llrr}
b_{\text {con } 11} & b_{\text {con } 12} & b_{\text {con } 13} & b_{\text {con } 14} \\
b_{\text {con } 21} & b_{\text {con } 22} & b_{\text {con } 23} & b_{\text {con } 24} \\
b_{\text {con } 31} & b_{\text {con } 32} & b_{\text {con } 33} & b_{\text {con } 34} \\
b_{\text {con } 41} & b_{\text {con } 42} & b_{\text {con } 43} & b_{\text {con } 44}
\end{array}\right]
$$

where, $b_{\text {conij }}$ is the knowledge contributor. If knowledge $K_{i}$ is contributed by medical person $M_{i}$, then the value of $b_{\text {conij }}$ is 1 ; otherwise, the value of $b_{\text {conij }}$ is 0 .

From the perspective of knowledge contribution, the closeness between two pieces of knowledge can be calculated by:

$$
\operatorname{sim}_{\mathrm{con}}\left(K_{i}, K_{j}\right)=\frac{\sum_{t=1}^{n}\left|\left(b_{\text {conit }}-b_{\text {conjt }}\right)\right|}{2}
$$

Knowledge modification matrix. If a piece of knowledge is modified by two medical persons, then the two medical persons are relatively close in knowledge relation. The modification frequency of knowledge by medical staff can be expressed as:

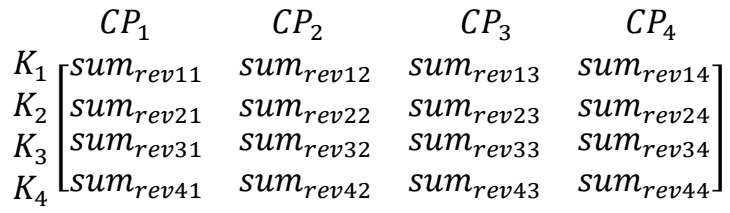

where, sum $_{\text {revij }}$ is an int about the modification frequency of knowledge $K_{i}$ by medical person $M_{i}$.

From the perspective of knowledge modification, the closeness between two pieces of knowledge can be calculated by:

$$
\operatorname{sim}_{r e v}\left(K_{i}, K_{j}\right)=\frac{\sum_{t=1}^{n} \mid \operatorname{sum}_{\text {rev it }}-\text { sum }_{\text {rev } j t} \mid}{n_{M}}
$$

Knowledge use matrix. If two pieces of knowledge are often used by the same medical person, their similarity distance is relatively small. The knowledge use of each medical person can be recorded as:

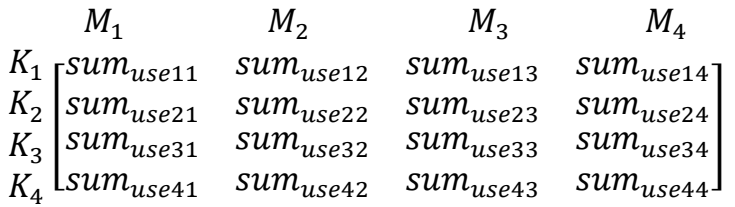

where, sum $_{\text {use } i j}$ is an int about the frequency of knowledge $K_{i}$ used by medical staff $M_{i}$.

From the perspective of knowledge use, the closeness between two pieces of knowledge can be calculated by:

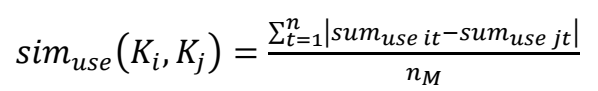

where, $n_{M}$ is the of number of contributors. If knowledge $K_{i}$ is contributed by medical person $M_{i}$, then $\operatorname{sum}_{u s e ~} i j=0$. In other words, the frequency of a medical person using the knowledge contributed by him/her is set to zero, eliminating the contributor's representations of his/her own knowledge $i$ and $j$.

(2) Calculation of the closeness between knowledge and medical staff

To measure the closeness between knowledge $(\mathrm{K})$ and medical staff $(\mathrm{M})$, the knowledge contributed or frequently used (top 10\%) was selected, and compared with the existing knowledge. That is to say, only knowledge modification and knowledge use are considered to compute the closeness between knowledge and medical staff.

From the perspective of knowledge source, the closeness between knowledge and medical staff can be computed by:

$$
\operatorname{sim}_{c p(K \& M)}\left(K_{a}, M_{b}\right)= \begin{cases}1 & \text { If } \mathrm{K}_{\mathrm{a}} \text { comes from } \mathrm{M}_{\mathrm{b}} \\ 0 & \end{cases}
$$

From the perspective of knowledge modification, the closeness between a piece of knowledge $K_{a}$ and a medical person $M_{b}$ can be computed by:

$$
\operatorname{sim}_{r e v(K \& M)}\left(K_{a}, M_{b}\right)=\frac{\sum_{x} \operatorname{sim}_{r e v}\left(K_{a}, K_{x}\right)}{n_{b}}
$$

where, knowledge $K_{x}$ is contributed by medical person $M_{b}$; $n_{b}$ is the times of contribution or use frequency by medical person $M_{b}$

From the perspective of knowledge use, the closeness between a piece of knowledge $K_{a}$ and a medical person $M_{b}$ can be computed by:

$$
\operatorname{sim}_{u s e(K \& M)}\left(K_{a}, M_{b}\right)=\frac{\sum_{x} \operatorname{sim}_{u s e}\left(K_{a}, K_{x}\right)}{n_{b}}
$$

where, knowledge $K_{x}$ is contributed by medical person $M_{b}$; $n_{b}$ is the times of contribution or use frequency by medical person $M_{b}$.

From the perspective of knowledge representation, the closeness between a piece of knowledge $K_{a}$ and a medical person $M_{b}$ can be computed by:

$$
\begin{gathered}
\operatorname{sim}_{\text {perfor }(K \& M)}\left(K_{a}, M_{b}\right)=\gamma_{1} \operatorname{sim}_{\operatorname{rev}(K \& M)}\left(K_{a}, M_{b}\right)+ \\
\gamma_{2} \operatorname{sim} \\
u s e(K \& M) \\
\left(K_{a}, M_{b}\right)
\end{gathered}
$$




$$
\sum_{i} \gamma_{i}=1
$$

To sum up, the closeness between a piece of knowledge $K_{a}$ and a medical person $M_{b}$ can be computed by:

$$
\begin{gathered}
\operatorname{sim}_{K \& M}\left(K_{a}, M_{b}\right)= \\
\delta_{1} \operatorname{sim}_{c p(K \& M)}\left(K_{a}, M_{b}\right)+\delta_{2} \operatorname{sim} \text { perfor }(K \& M) \\
\sum_{i} \delta_{1}=1
\end{gathered}
$$

\subsection{Patient-oriented smart service}

\subsubsection{Service content}

The patient-oriented smart service provides patients with the access to the proposed KMS-RTCMCPV. A patient can $\log$ on the system with his/her resident health card and input the TCM CPs he/she is interested in. With a distributed cloud storage structure, the patient-oriented smart service collects the HIS and EMR data from regional hospitals through the medical server, and provide the following functions for patients:

(1) Inquire patient information at any time
Each patient can input his/her number of resident health card via the patient-oriented smart service terminal to inquire about doctor advice, treatment progress and cost during the implementation of a CP.

(2) Obtain personalized service information

Some patients and their families are not aware of the importance of TCM CPs. Sometimes, they may refuse to receive the $\mathrm{CPs}$, causing $\mathrm{CPs}$ variation. Therefore, the $\mathrm{CP}$ knowledge should be pushed to them, making them understand the CPs. Based on the situation of each patient, our system pushes he knowledge of the TCM CPs related to his/her disease and the doctor advice to the patient. In addition, the patient's utilization rate of the pushed knowledge is collected to optimize the push service.

(3) Provide evaluation information

After ending the TCM CP, each patient can evaluate the $\mathrm{CP}$ process on mobile terminals. The evaluation information will not be made public, but will be collected regularly by the Evaluation Center. The Center will integrate the evaluations by all patients, and provide the integrated data to each hospital.

The operation of patient-oriented smart service is illustrated in Figure 7.

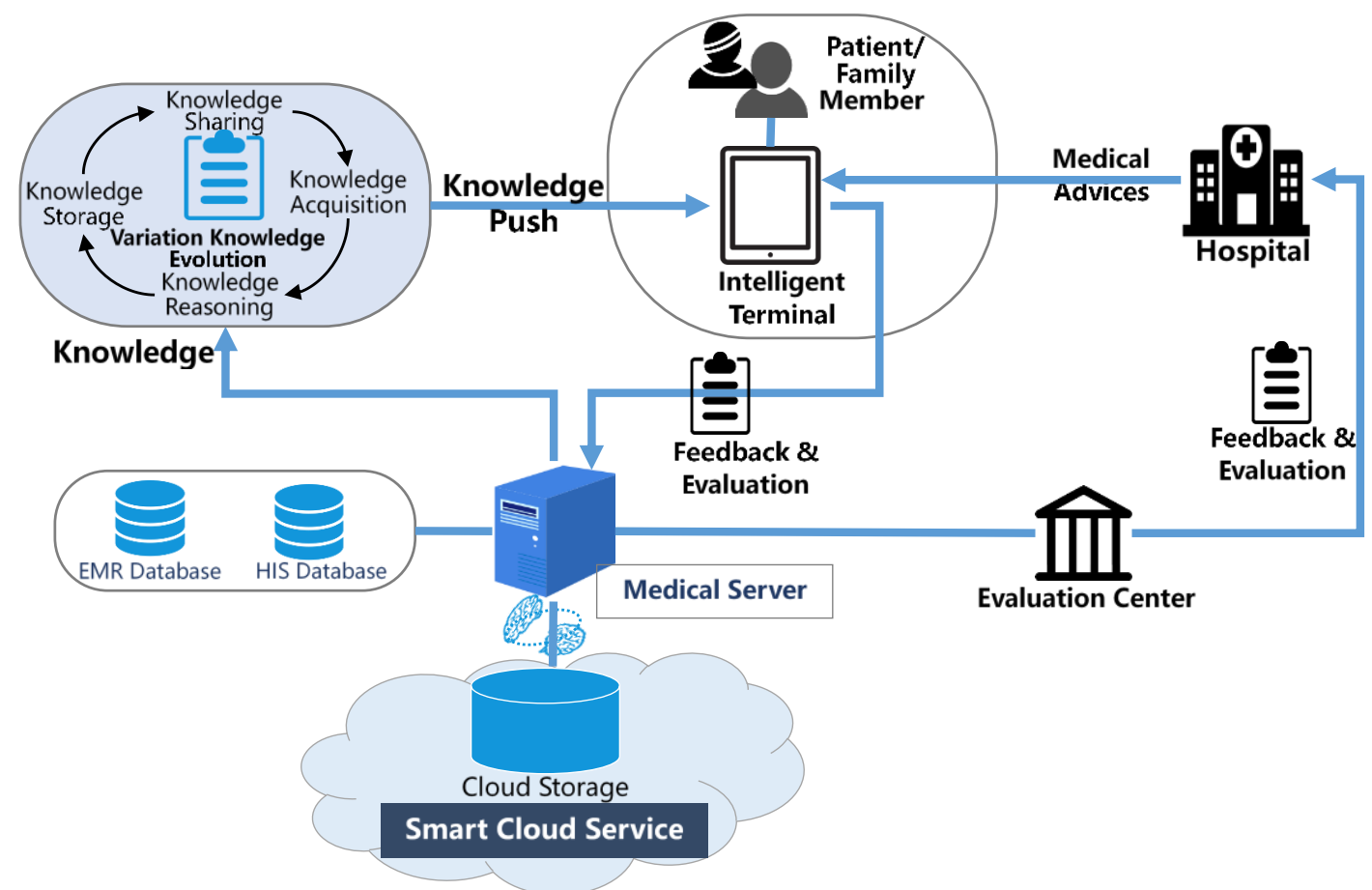

Figure 7. The operation of patient-oriented smart service

\subsubsection{Service implementation}

The CP knowledge should be pushed smartly to patients to enhance their awareness of TCM CPs and reduce the variation risk. The patients only receive knowledge without making any contribution or modification. Thus, patient-oriented smart service only considers the use of knowledge as the form of knowledge representation. The smart knowledge push is

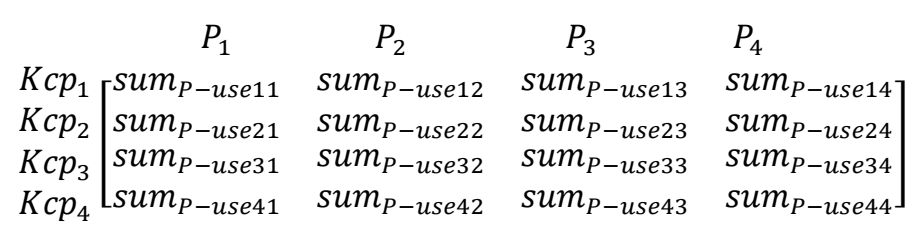

completed based on the closeness between the TCM CPs knowledge and patients.

(1) Matrix design

If two pieces of knowledge $K c p$ are often used by the same patient $P$, then their similarity distance is relatively small. The knowledge use by a patient can be recorded as:

$\left.\begin{array}{cl}P_{3} & P_{4} \\ \text { sum }_{P-u s e 13} & \text { sum }_{P-u s e 14} \\ \text { sum }_{P-u s e 23} & \text { sum }_{P-u s e 24} \\ \text { sum }_{P-u s e 33} & \text { sum }_{P-u s e 34} \\ \text { sum }_{P-u s e 43} & \text { sum }_{P-u s e 44}\end{array}\right]$


where, sum $_{P-u s e} i j$ is an int about the frequency of knowledge $K c p_{i}$ used by patient $P_{i}$. If knowledge $K c p_{i}$ is contributed by

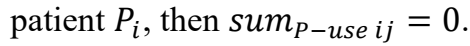

(2) Calculation of the closeness between knowledge and patient

To measure the closeness between knowledge $\left(K c p_{x}\right)$ and patient $\left(P_{b}\right)$, the knowledge frequently used (top 10\%) was selected. Then, the closeness $\operatorname{sim}_{K c p \& P}\left(K c p_{a}, P_{b}\right)$ between knowledge $K c p_{a}$ and patient $P_{b}$ can be computed by:

$$
\frac{\operatorname{sim}_{K c p \& P}\left(k_{a}, P_{b}\right)=\operatorname{sim}_{P-u s e(K c p \& P)}\left(k_{a}, P_{b}\right)=}{\frac{\sum x \operatorname{sim}_{P-u s e(K c p \& P)}\left(K c p_{a}, K c p_{x}\right)}{n_{b}}}
$$

where, $K c p_{a}$ is the knowledge not used by patient $P_{b} ; n_{b}$ is the number of pieces of knowledge contributed by patient $P_{b}$; $k c p_{x}$ is contributed by patient $P_{b}$. Our system automatically pushes a piece of knowledge to a patient if the knowledge is closely related to the patient.

\subsection{Smart cloud service}

The smart cloud service is the core of our system. In cloud storage, smart data mining and perception are achieved through machine learning. Here, cloud storage and intelligent mining are integrated to build a learning knowledge database. The information of all users, including doctors and patients, is continuously added to the system. Once training data are connected to the database, new data and rule database are connected. Then, the data are pushed to the relevant server nodes by the inference engine and interpretation tools.

The operation of the smart cloud service is explained in Figure 8.

To sort knowledge more effectively, the author designed a classification model of learned knowledge (Figure 9). When a user stores the collected data to the cloud, the data mining process and the classification rule of neural knowledge are displayed presented in the model.

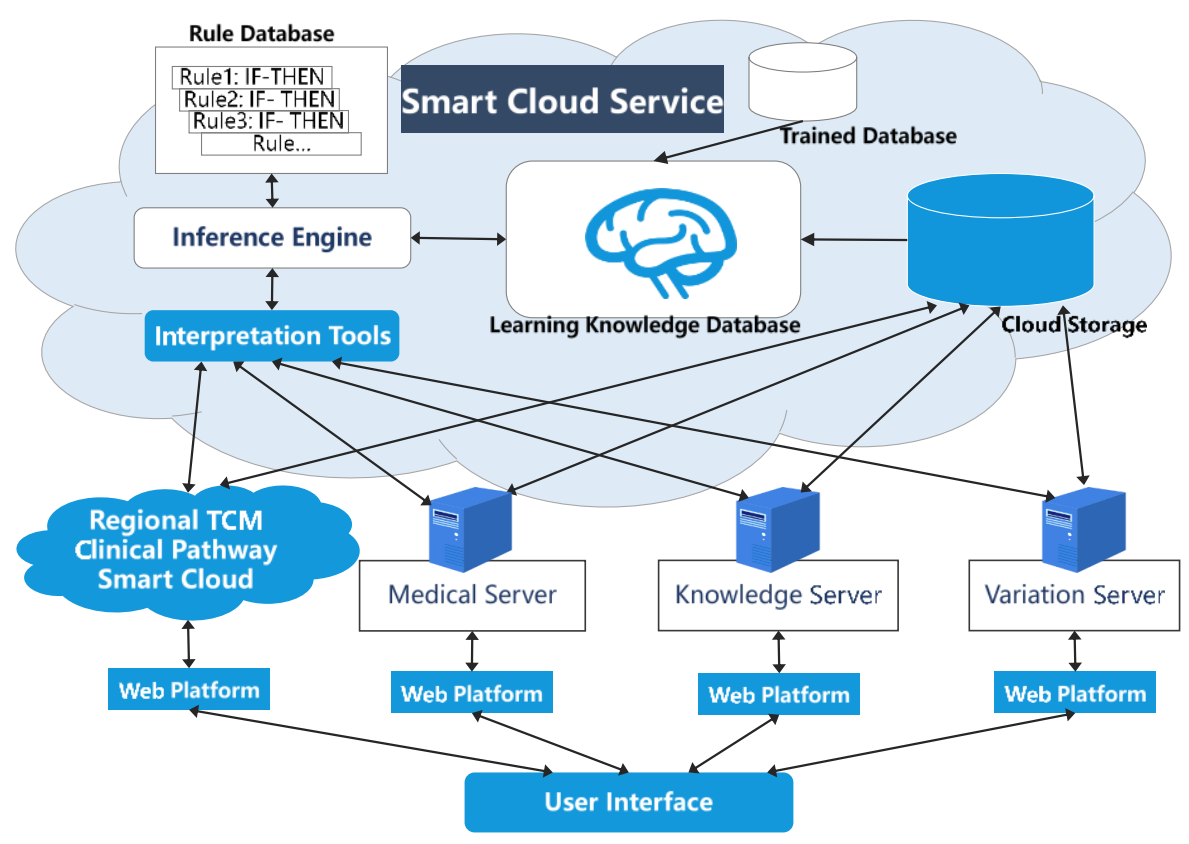

Figure 8. The operation of the smart cloud service

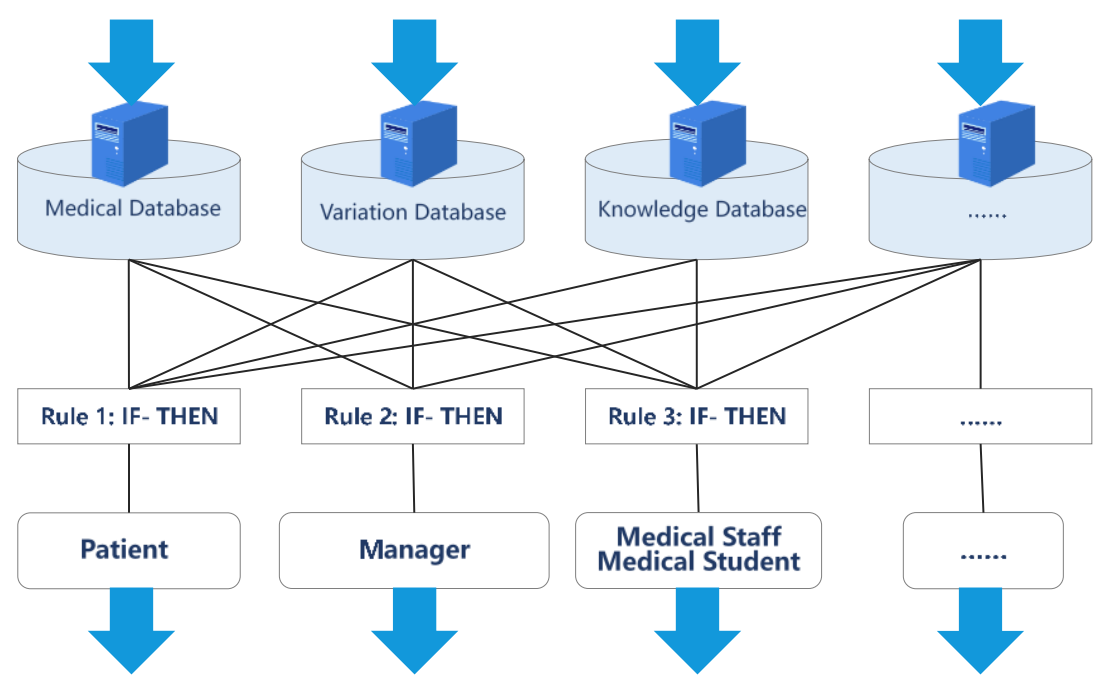

Figure 9. The classification model of learned knowledge 


\section{CONCLUSIONS}

The previous KMS-RTCMCPV has many problems, ranging from the lack of variation warning, the limited feedback channel, and the inability to share empirical knowledge. To solve the problems, this paper establishes a novel KMS-RTCMCPV based on cloud services. Through mining the regional medical data, the proposed system supports early warning of TCM CPs variation, and provides patients with a robust feedback mechanism and a smart knowledge push service. The system is realized after computing the closeness between TCM CPs knowledge, between knowledge and medical staff, and between knowledge and patients. Our system successfully enhances the diagnosis and treatment of Chinese medicine hospitals, and improves the patient satisfaction. The further research will combine the health records of regional residents with the proposed KMS-RTCMCPV, aiming to further optimize the knowledge management and process of regional TCM CPs.

\section{ACKNOWLEDGEMENT}

This research was supported by Zhejiang Provincial natural science foundation under Grant No.LY16G030009 and Zhejiang Provincial Philosophy and Social Science foundation under Grant No.16ZJQN049YB.

\section{REFERENCES}

[1] Lauck, S.B., Wood, D.A., Baumbusch, J., Kwon, J.Y., Stub, D., Achtem, L., Blanke, P., Boone, R.H., Cheung, A., Dvir, D., Gibson, J.A., Lee, B., Leipsic, J., Moss, R., Perlman, G., Polderman, J., Ramanathan, K., Ye, J., Webb, J.G. (2016). Vancouver transcatheter aortic valve replacement clinical pathway minimalist approach, standardized care, and discharge criteria to reduce length of stay. Circulation-Cardiovascular Quality and Outcomes, 9(3): 312-321. https://doi.org/10.1161/CIRCOUTCOMES.115.002541

[2] Kinsman, L., Rotter, T., James, E., Snow, P., Willis, J. (2010). What is a clinical pathway? development of a definition to inform the debate. BMC Medicine, 8(1): 3133. https://doi.org/10.1186/1741-7015-8-31

[3] Aarnoutse, M.F., Brinkkemper, S., de Mul, M., Askari, M. (2018). Pros and cons of clinical pathway software management: A qualitative study. Studies in Health Technology \& Informatics, 247: 526-530.

[4] Xu, W., Zhu, Y.X., Geng, Y.B. (2018). Development of an open metadata schema for clinical pathway (openCP) in China. Methods of Information in Medicine, 57(4): 159-167. https://doi.org/10.3414/ME17-01-0110

[5] Zhao, X., Wang, J., Chen, G. (2019). Development status and research of countermeasures of TCM clinical pathway. Chinese Hospital Management, 39(2): 44-46.

[6] Xu, W., Zhu, Y.X., Wang, X. (2017). An open metadata schema for clinical pathway (openCP) in China. Studies in Health Technology and Informatics, 245: 1344-1344.

[7] Zhao, Y.Q., Min, Z., Liang, Z.H., Cai, Y. (2014). Development of clinical pathway for stroke management: An e-Delphi survey. IEEE International Conference on Bioinformatics \& Biomedicine, 1: 328-329. https://doi.org/10.1109/BIBM.2013.6732706
[8] Sihoe, A.D.L., Yu, P.S.Y., Kam, T.H., Lee, S.Y., Liu, X.Y. (2016). Adherence to a clinical pathway for videoassisted thoracic surgery: Predictors and clinical importance. Innovations (Phila), 11(3): 179-186. https://doi.org/10.1097/imi.0000000000000279

[9] Du, Z.H., Sun, X.M. (2019). Clinical pathway for the community-level management of patients with type 2 diabetes. The International Journal of Health Planning and Management, 34(3): 975-985. https://doi.org/10.1002/hpm.2868

[10] Messing, B.P., Ward, E.C., Lazarus, C., Ryniak, K., Kim, M., Silinonte, J., Gold, D., Thompson, C.B., Pitman, K.T., Blanco, R., Sobel, R., Harrer, K., Ulmer, K., Neuner, G., Patel, K., Tang, M., Lee, G. (2019). Establishing a multidisciplinary head and neck clinical pathway: An implementation evaluation and audit of dysphagia-related services and outcomes. Dysphagia, 34(1): 89-104. https://doi.org/10.1007/s00455-0189917-4

[11] Buczak-Stec, E., Goryński, P., Nitsch-Osuch, A., Kanecki, K., Tyszko, P. (2017). The impact of introducing a new hospital financing system (DRGs) in Poland on hospitalisations for atherosclerosis: An interrupted time series analysis (2004-2012). Health Policy, 121(11): https://doi.org/10.1016/j.healthpol.2017.09.009

[12] Bayerstadler, A., Van Dijk, L., Winter, F. (2016). Bayesian multinomial latent variable modeling for fraud and abuse detection in health insurance. Insurance: Mathematics and Economics, 71: 244-252. https://doi.org/10.1016/j.insmatheco.2016.09.013

[13] Xu, X., Tao, J., Wei, Z.J., Wang, J.M. (2017). Incorporating topic assignment constraint and topic correlation limitation into clinical goal discovering for clinical pathway mining. Journal of Healthcare Engineering, 2017: 5208072. https://doi.org/10.1155/2017/5208072

[14] Lu, Y., Li, J. (2019). Efficient searchable public key encryption against keyword guessing attacks for cloudbased EMR systems. Cluster Computing, 22: 285-299. https://doi.org/10.1007/s10586-018-2855-y

[15] Gutenstein, M., Pickering, J.W., Than, M. (2019). Development of a digital clinical pathway for emergency medicine: Lessons from usability testing and implementation failure. Health Informatics Journal, 25(4): $1563-1571$ https://doi.org/10.1177/1460458218779099

[16] Huang, Z.X., Dong, W., Ji, L., He, C.H., Duan, H.L. (2016). Incorporating comorbidities into latent treatment pattern mining for clinical pathways. Journal of Biomedical Informatics, 59: 227-239. https://doi.org/10.1016/j.jbi.2015.12.012

[17] Yan, H., Van Gorp, P., Kaymak, U., Lu, X.D., Ji, L, Chiau, C.C., Korsten, H.H.M., Duan, H. (2018). Aligning event logs to task-time matrix clinical pathways in BPMN for variance analysis. IEEE Journal of Biomedical and Health Informatics, 22(2): 311-317. https://doi.org/10.1109/jbhi.2017.2753827

[18] Bultijnck, R., Van De Caveye, I., Rammant, E., Everaert, S., Lumen, N., Decaestecker, K., Fonteyne, V., Deforche B., Ost, P. (2018). Clinical pathway improves implementation of evidence-based strategies for the management of androgen deprivation therapy-induced 
side effects in prostate cancer patients. BJU International, 121(4): 610-618. https://doi.org/10.1111/bju.14086

[19] Chen, C.S., Liang, W.Y., Hsu, H.Y. (2014). A cloud computing platform for ERP applications. Applied Soft Computing, 27(C):

127-136. https://doi.org/10.1016/j.asoc.2014.11.009

[20] Hu, S., Zhou, L., Dong, N., Zhou, Y., Gao, Z.S., Xu, J., Liang, Z.W. (2017). The design and implementation of the privacy protection system of a Regional Health Information Platform. 2016 IEEE International Conference on Bioinformatics and Biomedicine (BIBM), 1 : 964-969. https://doi.org/10.1109/BIBM.2016.7822654

[21] Islam, M.M., Razzaque, M.A., Hassan, M.M., Ismail, W.N., Song, B. (2017). Mobile cloud-based big healthcare data processing in smart cities. IEEE Access, 5: 11887-11899. https://doi.org/10.1109/access.2017.2707439

[22] Kaur, P. D., Chana, I. (2014). Cloud based intelligent system for delivering health care as a service. Computer Methods and Programs in Biomedicine, 113(1): 346-359. https://doi.org/10.1016/j.cmpb.2013.09.013
[23] Toy, J.M., Drechsler, A., Waters, R.C. (2018). Clinical pathways for primary care: Current use, interest and perceived usability. Journal of the American Medical Informatics Association, 25(7): 901-906. https://doi.org/10.1093/jamia/ocy010

[24] Li, K., Yang, H., Zhang, Y.L., Zhu, Y., Xin, Y., Meng, Q. (2015). Improving public health multidimensional services through the use of smart cloud model. International Journal of Smart Home, 9(8): 205-218. https://doi.org/10.14257/ijsh.2015.9.8.22

[25] Ciagli, E., Antonuzzo, L., Frosini, F., Cocchi, D., Regolini, J., Benassi, A., Tortoli, P., Belardinelli, A., Costanzo, F.D., Iadanza, E. (2017). Analysis and optimization of clinical pathway of a cancer patient in a University Hospital. In EMBEC \& NBC 2017, Springer, Singapore, pp. 715-718. https://doi.org/10.1007/978981-10-5122-7_179

[26] Kasmire, K.E., Hoppa, E.C., Patel, P.P., Boch, K.N., Sacco, T., Waynik, I.Y. (2019). Reducing invasive care for low-risk febrile infants through implementation of a clinical pathway. Pediatrics, 143(3): e20181610. https://doi.org/10.1542/peds.2018-1610 